\title{
Projection Operators Technique in the Theory of Open Quantum Systems
}

\author{
V. Semin ${ }^{\mathrm{a}}$ and F.Petruccione \\ University of KwaZulu-Natal and National Institute for Theoretical Physics (NITheP), Quantum Research Group, \\ School of Chemistry and Physics, Westville, Durban, KwaZulu-Natal, 4001, South Africa
}

\begin{abstract}
In this work we study various aspects of the projection operators technique and application of projection operators to description of open quantum systems.
\end{abstract}

The Nakajima-Zwanzig master equation [1,2] is widely used in various applications [3]. In fact, the Nakajima-Zwanzig master equation is no more then the Liouville equation, rewritten for some relevant part of a density operator, and the relevant part of the total density operator is extracted by some specific projection operator. From mathematical point of view, the Nakajima-Zwanzig equation represents a very ponderous integro-differential equation, that is extremely difficult to study in its general form. Despite this fact, the form of the equation is convenient for systematic application of the perturbation theory. By truncating a perturbation series on some term and by using the different simplifications and assumptions, one can derive the majority of known master equations.

An alternative to the Nakajima-Zwanzig master equation may be, so called, time-convolutionless master equation [4]. In any order of the perturbation theory, the given equation is no more then the ordinary differential equation with coefficients, depending on time in a complex manner. The main feature of these generalized equations is the independence of its abstract form from the concrete choice of the projection operator, which can be used for simultaneously investigation of a broad class of the various forms of the kinetic equations.

In this work we investigate the generalized master equations, derived with the help of different projection operators, and apply them to describe open quantum systems. In particular, we show [5] that using some ideas of non-equilibrium thermodynamics, one can derive the non-linear master equations, connecting the dynamic and thermodynamic characteristics of an open system. On the other hand, we will show a special class of projection operators, that are always leads to an integrable in quadratures master equation in any order of the perturbation theory.

\section{References}

1. R. Zwanzig, J. Chem. Phys. 33, 1338-1341 (1960)

2. S. Nakajima, Prog. Theor. Phys. 20, 948-959 (1958)

3. H.-P. Breuer and F. Petruccione, The Theory of Open Quantum Systems (Oxford, 2002)

4. S. Chaturvedi, F. Shibata, Z. Phys. B 35, 297-308 (1979)

5. V. Semin, F. Petruccione, Phys. Rev. A 90, 052112 (2014)

${ }^{a}$ Corresponding author: veyvlet@mail.ru 